九州大学学術情報リポジトリ

Kyushu University Institutional Repository

\title{
An Age-Period-Cohort Analysis of Prevalence and Consultation Rate for Dyslipidemia in Japan
}

Okui, asuku

Kyushu University Hospital

http://hdl. handle. net/2324/4149944

出版情報: Asia- Pacific journal of public health, pp.1-7, 2020-10-08. Asia-Pacific Academic Consortium for Public Health Science Press

バージョン :

権利関係 : 


\begin{abstract}
This study involved an age-period-cohort (APC) analysis of the consultation rate and prevalence of dyslipidemia in Japan, based on Patient Survey data from 1999 to 2017 and open data of national database of health insurance claims and specific health checkups in Japan from 2013 to 2016. Our results showed that the consultation rates were lower than the prevalence, regardless of age, year, and sex, and particularly among middle-aged and male respondents. Additionally, both the consultation rate and prevalence increased with increasing age to a greater extent among women than men, and the degree of increase in the consultation rate was larger than that in prevalence among women. Furthermore, although the cohort effect on prevalence began to decrease among men in cohorts born in approximately 1960 , the effect decreased among women in cohorts born between the 1930s and 1960s and exhibited an increasing trend thereafter.
\end{abstract}

Keywords: age-period-cohort analysis, dyslipidemia, Japan, gender-analysis, the Patient Survey, the NDB Open data 


\section{What We Already Know}

- $\quad$ According to the Patient Survey, the estimated numbers of both male and female patients in Japan increased from 1999 to 2017.

- $\quad$ According to the National Health and Nutrition Survey in Japan, certain subpopulations were untreated despite a suspicion of dyslipidemia, and treatment rates were lower in younger age groups.

- $\quad$ No previous studies conducted an age-period-cohort analysis for trends in the dyslipidemia prevalence or consultation rate in Japan, and these trends have not yet been investigated over ages, periods and birth cohorts.

\section{What This Study Adds}

- $\quad$ The dyslipidemia consultation rates were lower than the prevalence in all cases, and more than half of patients with dyslipidemia aged $40-74$ years were untreated, regardless of age and sex.

- $\quad$ Both the consultation rate and prevalence increased with increasing age, particularly among women, and the degree of increase in the consultation rate was larger than that in the prevalence among women.

- $\quad$ Although the cohort effect on prevalence among men began to decrease in cohorts born in approximately 1960, the effect among women decreased in cohorts born between the 1930s and 1960s and tended to increase thereafter.

\section{Introduction}


The number of Japanese individuals with dyslipidemia, one of many lifestyle-related diseases, has continued to increase, ${ }^{1}$ with estimates showing 1.14 million patients with dyslipidemia in 1999 — a number that has since increased to 2.21 million. The diagnostic criteria of dyslipidemia are as follows: low-density lipoprotein (LDL) cholesterol value exceeds $140 \mathrm{mg} / \mathrm{dl}$; high-density lipoprotein (HDL) cholesterol is below $40 \mathrm{mg} / \mathrm{dl}$; triglyceride value exceeds $150 \mathrm{mg} / \mathrm{dl}^{2}$ If either of the criterion is met, the diagnosis of dyslipidemia is attached. Given that dyslipidemia can lead to arteriosclerosis, metabolic syndrome, or other lifestyle-related diseases ${ }^{2}$ its prevention has continued to be an important public health issue. Although several studies across Japan have investigated dyslipidemia prevalence, ${ }^{3}$ fewer epidemiological studies have been available compared to other lifestyle-related diseases, such as diabetes or hypertension. Moreover, birth cohort effects have yet to be investigated, while trends in cohort effects have remained unknown. As the Japanese lifestyle changes with birth cohorts, cohort effects on prevalence also change. Although a larger increase in the estimated number of women than men has been observed in Japan, ${ }^{1}$ whether differences in birth cohort effects by sex exist have remained unknown.

Age-period-cohort analysis (APC) has often been used as an analytical method for investigating disease trends. ${ }^{4}$ APC analysis is specifically used in the public health domain to analyze trends in the incidence or mortality of a disease, categorizing changes in statistics into age, period, and cohort effects. This method has also been used to investigate mortality rates within representative cancer subtypes or cardiovascular diseases in Japan., ${ }^{5,6}$ Furthermore, the Bayesian APC analysis model allows the estimation of not only age, period, and cohort effects by sex but also female/male prevalence ratios on each effects. Notably, the lack of APC analysis for dyslipidemia has been apparent in not only Japan but also other countries. 
The Patient Survey in Japan is conducted to investigate the number of patients receiving treatment for a disease, and the data can be used to obtain the estimated number of patients with the disease ${ }^{1}$. By applying an APC analysis to the Patient Survey data, we could evaluate trends in the rate of hospital consultation for dyslipidemia. However, certain subpopulations are known to remain untreated, despite a suspicion of dyslipidemia. ${ }^{7}$ Therefore, in addition to the Patient Survey in Japan, we analyzed a national database of health insurance claims and specific health checkups of Japan (NDB) open data. ${ }^{8}$ The specific health checkups include measurements of laboratory test values related to lifestyle-related diseases ${ }^{9}$; therefore, by using the NDB Open data, we could infer the prevalence of dyslipidemia in recent years. Additionally, an analysis of both the Patient Survey and NDB Open data would enable a better understanding of the difference between the consultation rate and prevalence of dyslipidemia in Japan.

The present study analyzed trends in the prevalence and consultation rate of dyslipidemia using APC analysis of data collected by the Patient Survey and the NDB Open data in Japan.

\section{Methods}

Data from the Patient Survey ${ }^{1}$, NDB Open data $^{8}$, and Vital Statistics ${ }^{10}$ in Japan were used for analysis. The Patient Survey and Vital Statistics data were used to calculate the consultation rate of dyslipidemia. The Patient Survey, which is conducted once every 3 years, tallies the number of patients who visit a hospital or clinic at sites throughout Japan. Hospitals and clinics surveyed are randomly selected, except for those having $>500$ beds, which are definitely included. Medical institutes across Japan are stratified based on strata, such as the region and type of the institute (e.g., hospital, general clinic, or dental clinic), and 
stratified random sampling is used to select the survey subjects. ${ }^{11}$ Specifically, as the strata for stratified random sampling for the hospitals, region, institute type, and bed size are used. Also, as the strata for general clinics, prefectures, main medical subjects, and bed status (present/absent) are used. Data for the estimated total number of patients from the seven Patient Surveys conducted from 1999 to 2017 were used. Patients with dyslipidemia were defined as those who received an ICD10 code of E78. Although the estimated number of dyslipidemia patients for 1996 is published, the values of some age groups cannot be obtained. Therefore, we used the data from 1999. The estimated total number of patients was calculated based on the number of outpatients and hospitalized patients in the survey. ${ }^{1}$ Population statistics data for each sex and age group was obtained from Vital Statistics. ${ }^{10} \mathrm{We}$ used data that were available in public, and an ethical approval is not needed for this study.

Next, we used the NDB Open data to calculate the prevalence of dyslipidemia. The NDB Open data contain information from specific health checkups in Japan. ${ }^{8}$ This data set was also used in a previous study. ${ }^{12}$ The specific health checkups began in 2008 with the aim of preventing the development of lifestyle-related diseases. The subjects of these checkups were health insurance participants aged 40-74 years, and the subjects were encouraged to participate in the checkups every year. ${ }^{9}$ The examinations included laboratory testing, and the aggregated laboratory test values from 2013 to 2016 are now publicly available. The details of the specific health checkups have been reported elsewhere. ${ }^{9,13,14}$ We used triglyceride data in this study, and the numbers of subjects in whom this parameter was measured per year were as follows: $2013,21,899,582 ; 2014,23,849,334 ; 2015,24,720,546$; and 2016, $25,074,510 .{ }^{15}$ A person was diagnosed with dyslipidemia if the triglyceride concentration exceeded $150 \mathrm{mg} / \mathrm{dl},{ }^{2}$ We calculated the prevalence of dyslipidemia in each year and sex 
using the ratio of the number of subjects whose triglyceride levels exceeded this cutoff to the total number of subjects.

As descriptive analysis, we calculated the consultation rates and prevalence of dyslipidemia for men and women according to periods and age groups. Then, the Bayesian APC model was used to simultaneously model the data for both sexes in order to calculate the female/male consultation rate and prevalence ratios in each age group, period, and cohort. For the APC analysis of the consultation rate based on the Patient Survey data, let $y_{i j r}$ as the estimated number of patients for the age group $i(1, \ldots, I)$ in period $j(1, \ldots, J)$ of sex $r(1,2)$. In the model, $y_{i j r}$ are assumed to follow the following Poisson distribution whose mean are $\lambda_{i j r}$

$$
\begin{gathered}
y_{i j r} \sim \text { Poisson }\left(\lambda_{i j r}\right), \\
\log \left(\lambda_{i j r}\right)=\delta_{r}+\alpha_{i r}+\beta_{j r}+\gamma_{k r}+z_{i j r}+\log \left(n_{i j r}\right)
\end{gathered}
$$

, where $\delta_{r}$ are the intercepts by sex, $\alpha_{i r}$ are the effects of age groups by sex, $\beta_{j}$ are period effects by sex, $\gamma_{k}(k=1, ., K)$ are cohort effects by sex, $z_{i j}$ are random effects that are defined for each sex, period and age group, and $n_{i j r}$ are the corresponding population. For the identifiability of the parameters, the restriction that $\sum_{i=1}^{I} \alpha_{i r}=\sum_{j=1}^{J} \beta_{j r}=\sum_{k=1}^{K} \gamma_{k r}=0$ is put. $z_{i j r}$ are assumed to be generated from normal distribution whose mean is zero. For the priors of age, period, and cohort effects, first-order random walk was used to identify the parameters. Age groups were defined in 5-year units from 30-34 years to 85-89 years. The cohort used for APC analysis was assigned by 1-year shifts, that is, the generation aged 85 89 years in 1999 was identified as the first cohort, while the generation aged 30-34 years in 
2017 was identified as the last cohort. The Hamiltonian Monte Carlo method (http://mcstan.org/) was used to estimate the parameters of the APC model.

The above-described APC model was also used for the APC analysis of prevalence data based on the NDB Open data set. However, in this case, $y_{i j r}$ denoted the number of subjects whose triglyceride levels exceeded $150 \mathrm{mg} / \mathrm{dl}$, and $n_{i j r}$ denoted the corresponding total number of subjects. Additionally, age groups were defined in 5-year units from 40-44 to 7074 years. The cohort used for the APC analysis was assigned by 1-year shifts; specifically, the generation aged 70-74 years in 2013 was identified as the first cohort, while the generation aged 40-44 years in 2016 was identified as the last cohort.

All statistical analyses were conducted using R 3.6.3 software (https://www.R-project.org/).

\section{Results}

Table 1 shows the consultation rate and prevalence for dyslipidemia per 1,000 persons for each period and age group. Although the consultation rates continued to increase and decrease during the analyzed periods, increases in the consultation rates were evident in 50 or more years for men and women. There was a large difference between the consultation rate and prevalence, regardless of age group and sex. Although the prevalence tended to decrease among men with increasing age, beginning at 50 years, the opposite trend was observed among women.

Figure 1 shows the APC analysis results for the consultation rate for dyslipidemia based on the Patient Survey data. Accordingly, the age effect among men consistently increased with 
age and peaked at 75-79 years. The period effect for men remained stable from 1999 to 2008 but increased thereafter. The cohort effect for men increased until around cohort born in 1938 and remained stable until around cohort born in 1964. The age effect among women began to rapidly increase from 45-49 years and peaked at 75-79 years. The period effect among women remained stable from 1999 to 2005 but increased thereafter. The cohort effect among women increased until around cohort born in 1938, decreased thereafter, and remained stable from around cohort born in 1976. The female/male consultation rate ratio for age was below 1 until 45-49 years but exceeded 1 in subsequent ages. Although the ratio for period continued to increase and decrease, an overall increase in the ratio was observed from 1999 to 2017. The ratio for cohort showed a decreasing trend until cohort born in around 1964 but increased thereafter. Notably, the ratio remained above 1 throughout the analyzed cohorts.

Figure 2 shows the results of the APC analysis of dyslipidemia prevalence based on the NDB Open data. Although the female/male prevalence ratio also increased with increasing age, the ratio remained below 1 across all analyzed age groups. Despite subtle differences, the trends in the cohort effects were relatively similar to those in the consultation rates. The cohort effect for men began to decrease among cohorts born in approximately 1960 .

Although the cohort effect for women decreased in cohorts born approximately prior to the late 1960 s, this effect exhibited an increasing trend thereafter. Therefore, the female/male prevalence ratio tended to increase among cohorts born in the $1960 \mathrm{~s}$.

\section{Discussion}

Our results showed considerable differences in the consultation rates and prevalence of dyslipidemia between men and women. The prevalence was higher among men than women, 
and this was consistent with data from other eastern Asian countries (e.g., China). ${ }^{16-18}$ In Japan, a certain percentage of people are known to remain untreated despite a suspicion of dyslipidemia. ${ }^{7}$ However, the results of this study demonstrate that more than half of all people with dyslipidemia remained untreated even in older ages, and that this proportion was particularly large among men.

Regarding the estimated age effect of the APC analysis, the female/male consultation rate ratio increased to $>1$ among those aged $\geq 50$ years, whereas the prevalence ratio remained $<1$ across all age groups. The data suggest that both the dyslipidemia prevalence and treatment rate increased with increasing age, particularly among women. Furthermore, the period effects for the consultation rates exhibited increasing trends during the analyzed periods. As shown in Figure 1, the degree of increase in the period effects began to grow in 2008 . The consultation rate of dyslipidemia can be also determined from the Comprehensive Survey of Living Conditions in Japan. ${ }^{19}$ This nationwide survey investigates the consultation rates of major diseases in subjects selected by a stratified random sampling of all households in Japan, and an increase in the dyslipidemia consultation rate was also reported by the survey. ${ }^{19}$ However, it remains uncertain whether the prevalence also increased during the same periods. One possible reason for this increase in the consultation rate is the initiation of the specific health checkups in 2008; possibly, the treatment rates also increased among those whose laboratory test values were abnormal. Furthermore, subjects at a high risk of developing lifestyle-related diseases are encouraged to change their lifestyles during these health checkups, which might have affected their consultation behaviors. The period effects might also have been affected by the change in the diagnostic criteria for dyslipidemia or the introduction of guidelines. The guideline for Prevention of Atherosclerotic Cardiovascular Diseases was first published in 1997 and presented the diagnostic criteria of dyslipidemia. ${ }^{20}$ Therefore, public awareness of the criteria 
for dyslipidemia may have increased gradually since the end of the $20^{\text {th }}$ Century. Additionally, the diagnostic criteria for dyslipidemia were changed in the 2007 revision of the guideline for Prevention of Atherosclerotic Cardiovascular Diseases. ${ }^{20}$ Prior to 2007, a person was diagnosed with dyslipidemia if the total serum cholesterol level exceeded $220 \mathrm{mg} / \mathrm{dl}$; however, this criterion was removed in 2007 . Therefore, the number of patients should have decreased, and therefore this change is not considered to be related to the observed increasing trend.

Regarding the cohort effect, we observed different trends depending on sex. Cohort effects generally reflect trends in risk factors that are specific for each birth cohort, such as lifestyle factors. The prevalence of obesity is one possible factor that may be associated with these trends. According to a previous study, the cohort effects on obesity prevalence among men began to decrease in cohorts born in approximately 1962-1971. In contrast, the cohort effects among women began to decrease from approximately 1938 until approximately 1976, and increased thereafter. ${ }^{21}$ Obesity is a known risk factor for dyslipidemia in Japan and might be related to the trends in the cohort effects. ${ }^{22}$ Smoking is another strong risk factor for dyslipidemia, and this association was also observed in Japan. ${ }^{23,24}$ However, the smoking prevalence was shown to increase in cohorts of men born between the1930s and 1970s and in women born between the 1940s and 1970s. ${ }^{25}$ Therefore, other factors are considered to be related to the cohort effect on dyslipidemia prevalence among women.

Some limitations of the preset study are worth noting. Given that the Patient Survey estimates the total number of patients based on the number of outpatients and inpatients in a day, the numbers are not actual values. As such, it could be possible that fewer men than women visit a hospital, which would lead to the underestimation of male dyslipidemia patients. Also, the discrepancy between the prevalence and consultation rate observed in this study may be due to an underestimation of the number of subjects with dyslipidemia in the Patient Survey. 
According to the results of the Comprehensive Survey of Living Conditions in 2016, the consultation rates were also shown to increase with increasing age in both men and women. The consultation rates of dyslipidemia among those aged 40-44 years were 2.7 and 21.2 per 1,000 persons among men and 21.2 women, respectively. ${ }^{19}$ The consultation rates among men and women aged 70-74 years were 81.8 and 153.5 per 1,000 persons, respectively, and the consultation rates in older age groups differed considerably between the survey data sets. However, even if the data of the Comprehensive Survey of Living Conditions were more accurate than those of the Patient Survey, the proportion of untreated persons remained larger than that of untreated persons among men and among the middle ages. Additionally, although dyslipidemia diagnoses were based on triglyceride concentration data, the ability to consider subjects whose LDL-C or HDL-C values were abnormal would lead to further increases in dyslipidemia prevalence than the values calculated in this study. As another limitation of the Patient Survey, considering that the survey uses the insurance names assigned to diseases, patients who diagnosed with dyslipidemia may not necessarily have dyslipidemia. Regarding the NDB Open data, the rate of subjects in the target population who were examined in specific health checkups was approximately 50\%, despite annual variances. Therefore, the nonparticipation of approximately half of the target population each year may have affected the analysis of dyslipidemia prevalence. Other available data, such as the National Health and Nutrition Survey, can be used to determine the dyslipidemia prevalence in Japan. ${ }^{7}$ However, the sample size of that study is overwhelmingly smaller than that of the NDB Open data set. Hence, to more accurately estimate the prevalence of dyslipidemia in Japan, a more large-scale epidemiological study is needed.

Finally, the results of this study suggest that the dyslipidemia consultation rates were generally lower than the prevalence. The association between the dyslipidemia prevalence and 
cardiovascular disease (CVD) is well known not only in Japan, but also in other Asian countries.

${ }^{26,27}$ Studies of Asia-Pacific countries have demonstrated low dyslipidemia treatment rates. ${ }^{28-30}$ It is important to assess the dyslipidemia treatment and consultation rates and the prevalence in other Asia-Pacific countries to prevent the progression of this condition and CVD. Assessments of the consultation rate and prevalence can enable us to identify the population among whom public awareness about treatment is particularly needed.

\section{Conclusion}

Overall, our results demonstrate that the dyslipidemia consultation rate was generally lower than the prevalence, particularly among middle-aged and male subjects. Both the prevalence and consultation rate increased with increasing age, particularly among women, and the degree of increase in the consultation rate was larger than the degree of increase in prevalence among women. Furthermore, although the cohort effect on prevalence among men began to decrease in cohorts born in approximately 1960, the cohort effect among women $\mathrm{d}$ decreased among women in cohorts born between the 1930s and 1960s and exhibited an increasing trend thereafter.

\section{Declaration of Conflicting Interests}

The authors declare that there is no conflict of interest.

\section{References}


1. Ministry of Health, Labour and Welfare. The Patient Survey. https://www.estat.go.jp/stat-search/files?page $=1 \&$ toukei $=00450022 \&$ tstat $=000001031167$. Accessed August 10, 2020.

2. Kinoshita M, Yokote K, Arai H, et al. Japan Atherosclerosis Society (JAS) Guidelines for Prevention of Atherosclerotic Cardiovascular Diseases 2017. J Atheroscler Thromb. 2018; 25(9): 846-984.

3. Okamura T. Dyslipidemia and cardiovascular disease: a series of epidemiologic studies in Japanese populations. J Epidemiol. 2010; 20(4) :259-265.

4. Smith TR, Wakefield J. A Review and Comparison of Age-Period-Cohort Models for Cancer Incidence. Stat Sci. 2016; 31(4):591-610.

5. Ito Y, Ioka A, Nakayama T, Tsukuma H, Nakamura T. Comparison of Trends in Cancer Incidence and Mortality in Osaka, Japan, Using an Age-Period-Cohort Model. Asian Pac J Cancer Prev. 2011; 12(4):879-888.

6. Ma E, Takahashi H, Mizuno A, Okada M, Yamagishi K, Iso H. Stratified ageperiod-cohort analysis of stroke mortality in Japan, 1960 to 2000. J Stroke Cerebrovasc Dis. 2007;16(3):91-102.

7. Ministry of Health, Labour and Welfare. The National Health and Nutrition Survey https://www.e-stat.go.jp/stat-search/files?page $=1 \&$ toukei $=00450171 \&$ tstat $=$ 000001041744. Accessed August 10, 2020. 
8. Ministry of Health, Labour and Welfare. the NDB Open data.

https://www.mhlw.go.jp/stf/seisakunitsuite/bunya/0000177182.html. Accessed August $10,2020$.

9. Ministry of Health, Labor and Welfare. Regarding specific medical examination and specific health guidance. http://www.mhlw.go.jp/bunya/shakaihosho/iryouseido01 /info02a.html. Accessed August 10, 2020.

10. Ministry of Health, Labour and Welfare. The Vital Statistics. https://www.estat.go.jp/statsearch/files?page $=1 \&$ layout $=$ datalist $\&$ toukei $=00450011 \&$ tstat $=000001028897 \&$ cycle $=7$ $\&$ tclass $1=000001053058 \&$ tclass $2=000001053061 \&$ tclass $3=000001053072$ Accessed August 10, 2020.

11. Ministry of Health, Labour and Welfare. Frequently asked questions about the Patient Survey. https://www.mhlw.go.jp/toukei/list/dl/10-20qa.pdf Accessed August $10,2020$.

12. Itoh H, Saito T, Nojiri S, Hiratsuka Y, Yokoyama K. National burden of the pharmaceutical cost of wet compresses and its cost predictors: nationwide crosssectional study in Japan. Health Econ Rev. 2019;9(1):20.

13. Fukasawa T, Tanemura N, Kimura S, Urushihara H. Utility of a Specific Health Checkup Database Containing Lifestyle Behaviors and Lifestyle Diseases for Employee Health Insurance in Japan. J Epidemiol. 2020;30(2):57-66. 
14. Tsushita K, S Hosler A, Miura K, et al. Rationale and Descriptive Analysis of Specific Health Guidance: the Nationwide Lifestyle Intervention Program Targeting Metabolic Syndrome in Japan. J Atheroscler Thromb. 2018;25(4):308-322.

15. Ministry of Health, Labor and Welfare. The data of specific health checkups and specific health guidances. https://www.mhlw.go.jp/bunya/shakaihosho/iryouseido01 /info02a-2.html. Accessed August 10, 2020.

16. Pan $\mathrm{L}, \mathrm{Yang} \mathrm{Z}, \mathrm{Wu} \mathrm{Y}$, et al. The prevalence, awareness, treatment and control of dyslipidemia among adults in China. Atherosclerosis. 2016; 248:2-9.

17. Liu X, Yu S, Mao Z, et al. Dyslipidemia prevalence, awareness, treatment, control, and risk factors in Chinese rural population: the Henan rural cohort study. Lipids Health Dis. 2018;17(1):119.

18. Cai L, Zhang L, Liu A, Li S, Wang P. Prevalence, awareness, treatment, and control of dyslipidemia among adults in Beijing, China. J Atheroscler Thromb. 2012;19(2):159_ 168.

19. Ministry of Health, Labour and Welfare. The Comprehensive Survey of Living Conditions. https://www.e-stat.go.jp/stat-search/files?page=1\&toukei=00450061. Accessed August 10, 2020.

20. Japan Atherosclerosis Society. Japan Atherosclerosis Society (JAS) Guidelines for Prevention of Atherosclerotic Cardiovascular Diseases 2007. http://www.jathero.org/publications/pdf/guideline_summary.pdf. Accessed August 10, 2020. 
21. Yamakita M, Uchida H, Kawamura K, Homma T, Odagiri Y. [Effects of Age, Period, and Cohort on the Trends in Obesity Rate and Energy Intake Ratio From Fat in Japanese Adults.] Nihon Koshu Eisei Zasshi. 2014; 61(8): 371-384.

22. Kuwabara M, Kuwabara R, Niwa K, et al. Different Risk for Hypertension, Diabetes, Dyslipidemia, and Hyperuricemia According to Level of Body Mass Index in Japanese and American Subjects. Nutrients. 2018;10(8):1011.

23. Ueyama C, Horibe $\mathrm{H}$, Yamase $\mathrm{Y}$, et al. Association of smoking with prevalence of common diseases and metabolic abnormalities in community-dwelling Japanese individuals. Biomed Rep. 2017;7(5):429-438.

24. Lee MH, Ahn SV, Hur NW, Choi DP, Kim HC, Suh I. Gender differences in the association between smoking and dyslipidemia: 2005 Korean National Health and Nutrition Examination Survey. Clin Chim Acta. 2011;412(17-18):1600-1605.

25. Okui T. Age-Period-Cohort Analysis of Healthy Lifestyle Behaviors Using the National Health and Nutrition Survey in Japan. J Prev Med Public Health. 2020;53(6). In press.

26. Ohira T, Iso H. Cardiovascular disease epidemiology in Asia: an overview. Circ J. 2013;77(7):1646-1652.

27. Lee J, Son H, Ryu OH. Management Status of Cardiovascular Disease Risk Factors for Dyslipidemia among Korean Adults. Yonsei Med J. 2017;58(2):326-338. 
28. Boo S, Yoon YJ, Oh H. Evaluating the prevalence, awareness, and control of hypertension, diabetes, and dyslipidemia in Korea using the NHIS-NSC database: A cross-sectional analysis. Medicine (Baltimore). 2018;97(51):e13713.

29. Aekplakorn W, Taneepanichskul S, Kessomboon P, et al. Prevalence of Dyslipidemia and Management in the Thai Population, National Health Examination Survey IV, 2009. J Lipids. 2014;2014: 249584.

30. Lin CF, Chang YH, Chien SC, Lin YH, Yeh HY. Epidemiology of Dyslipidemia in the Asia Pacific Region. International Journal of Gerontology. 2018;12(1):2-6. 


\section{Figure legends}

Figure 1: The APC analysis results for the consultation rate for dyslipidemia based on the Patient Survey data

The first line of the graph shows the age, period, and cohort effects on dyslipidemia prevalence for men, while the second line shows those for women. The third line of the graph shows the female/male consultation rate ratio for age, period, and cohort. Solid lines indicate estimates of each effect, while shadings show $95 \%$ credible intervals of each effect. Estimates of each point are connected for visualization.

Figure 2: The results of the APC analysis of dyslipidemia prevalence based on the NDB Open data

The first line of the graph shows the age, period, and cohort effects on dyslipidemia prevalence for men, while the second line shows those for women. The third line of the graph shows the female/male prevalence ratio for age, period, and cohort. Solid lines indicate estimates of each effect, while shadings show $95 \%$ credible intervals of each effect. Estimates of each point are connected for visualization. 
Table

Table 1. Consultation rate and prevalence for dyslipidemia per 1,000 persons for each period and age group

\begin{tabular}{|c|c|c|c|c|c|c|c|c|c|c|c|c|}
\hline $\begin{array}{l}\text { Data, sex and } \\
\text { Periods, age } \\
\text { groups }\end{array}$ & $30-34$ & $35-39$ & $40-44$ & $45-49$ & $50-54$ & $55-59$ & $60-64$ & $65-69$ & $70-74$ & $75-79$ & $80-84$ & $85-89$ \\
\hline \multicolumn{13}{|l|}{$\begin{array}{c}\text { Consultation rate } \\
\text { Men }\end{array}$} \\
\hline 1999 & 1.9 & 4.1 & 5.1 & 7.7 & 9.0 & 10.8 & 11.4 & 14.1 & 13.6 & 14.2 & 9.3 & 6.7 \\
\hline 2002 & 1.3 & 3.9 & 6.2 & 8.2 & 12.0 & 13.0 & 15.6 & 14.4 & 16.0 & 16.1 & 11.2 & 5.9 \\
\hline 2005 & 1.0 & 2.1 & 6.0 & 9.4 & 8.9 & 12.2 & 14.0 & 20.0 & 19.1 & 16.4 & 14.7 & 10.8 \\
\hline 2008 & 0.7 & 2.9 & 5.8 & 7.8 & 9.3 & 11.4 & 12.0 & 15.4 & 18.4 & 18.3 & 15.4 & 9.3 \\
\hline 2011 & 1.7 & 3.9 & 6.7 & 11.6 & 11.4 & 10.5 & 14.6 & 20.9 & 22.6 & 20.6 & 19.9 & 9.7 \\
\hline 2014 & 1.4 & 3.9 & 6.7 & 7.2 & 13.2 & 17.0 & 19.4 & 20.0 & 24.0 & 25.4 & 18.1 & 18.5 \\
\hline 2017 & 1.1 & 2.6 & 5.1 & 8.3 & 12.8 & 17.3 & 20.7 & 21.8 & 26.0 & 26.0 & 23.3 & 23.9 \\
\hline \multicolumn{13}{|l|}{ Women } \\
\hline 1999 & 0.7 & 1.6 & 2.6 & 6.0 & 14.6 & 29.3 & 38.2 & 42.9 & 40.0 & 35.4 & 16.9 & 9.9 \\
\hline 2002 & 0.7 & 1.0 & 3.4 & 6.2 & 15.3 & 30.9 & 45.1 & 49.2 & 51.3 & 38.8 & 27.3 & 15.8 \\
\hline 2005 & 0.4 & 0.9 & 2.3 & 4.5 & 16.0 & 27.1 & 44.5 & 57.7 & 56.2 & 46.3 & 31.5 & 17.8 \\
\hline 2008 & 0.7 & 1.3 & 2.9 & 6.0 & 11.1 & 25.3 & 35.5 & 47.4 & 49.4 & 45.3 & 33.0 & 22.3 \\
\hline 2011 & 0.8 & 1.3 & 3.1 & 5.4 & 16.7 & 29.9 & 47.0 & 62.7 & 61.4 & 60.0 & 44.8 & 27.3 \\
\hline 2014 & 0.8 & 1.0 & 3.4 & 6.2 & 16.7 & 38.3 & 49.8 & 58.3 & 68.2 & 59.1 & 47.0 & 30.1 \\
\hline 2017 & 0.9 & 1.3 & 3.1 & 5.7 & 17.8 & 31.2 & 47.7 & 55.3 & 74.4 & 69.7 & 54.7 & 39.7 \\
\hline \multicolumn{13}{|l|}{ Prevalence } \\
\hline 2013 & & & 284.1 & 307.7 & 310.1 & 294.3 & 276.9 & 260.0 & 235.3 & & & \\
\hline 2014 & & & 277.3 & 300.0 & 305.9 & 294.1 & 277.7 & 263.1 & 234.7 & & & \\
\hline 2015 & & & 272.0 & 294.1 & 301.1 & 291.0 & 277.3 & 262.4 & 233.1 & & & \\
\hline 2016 & & & 270.0 & 291.9 & 299.7 & 290.9 & 278.0 & 264.8 & 237.9 & & & \\
\hline \multicolumn{13}{|l|}{ Women } \\
\hline 2013 & & & 58.5 & 75.9 & 107.4 & 134.2 & 158.9 & 169.5 & 173.6 & & & \\
\hline 2014 & & & 57.0 & 72.8 & 102.4 & 129.0 & 153.7 & 167.5 & 169.5 & & & \\
\hline 2015 & & & 57.0 & 72.5 & 101.7 & 126.7 & 149.9 & 165.6 & 167.5 & & & \\
\hline 2016 & & & 57.9 & 74.2 & 102.8 & 126.6 & 150.1 & 168.3 & 170.2 & & & \\
\hline
\end{tabular}

